\title{
Reconstrucción mamaria con colgajo TRAM pediculado*
}

\author{
Drs. MAURICIO IBÁÑEZ R. ${ }^{1}$, GLADYS IBÁÑEZ R. ${ }^{1,2}$, NICOLÁS PEREIRA C. ${ }^{3}$, \\ Ints. CARLOS MANDIOLA B. ${ }^{4}$, ROMINA ANDINO N. ${ }^{4}$. \\ 1 Unidad de Cirugía Plástica, Clínica Dávila. \\ 2 Unidad de Patología Mamaria - Complejo Hospitalario San José. \\ Departamento de Cirugía, Hospital Clínico Universidad de Chile. \\ 4 Internos Facultad de Medicina Universidad de Chile. \\ Santiago, Chile.
}

Abstract

\section{Breast reconstruction with pedicled TRAM flap}

The pedicle transverse rectus abdominus myocutaneous flap (TRAM) is widely used for breast reconstruction, however is not exempt from complications. Between 2004 and 2010 the authors performed breast reconstruction after total mastectomy in 52 patients with pedicled TRAM flaps. Purpose: To describe the demography, outcomes and complications in patients operated for breast cancer and reconstructed with TRAM flap. To assess the influence of smoking, the laterality and timing of reconstruction. Material and Method: Retrospective, observational and comparative. We reviewed the medical records of 52 patients operated by the authors between 2004 and 2010. Four cases were excluded due to incomplete data. Results: In the 48 patients studied were 50 TRAM flaps. The most frequent comorbidity was smoking $(27 \%)$. Regarding the timing of reconstruction, 74\% (37) was performed immediately post-mastectomy and $26 \%$ (13) was deferred. Was used by $78 \%$ (39) ipsilateral TRAM, 18\% (9) contralateral and 4\% (2) bilateral. Only minor complications were observed and the cosmetic result evaluated by the surgeon's satisfaction was very good or excellent in $62 \%$ (31). Smoking, timing of reconstruction and laterality did not affect in complications or cosmetic outcome $(\mathrm{p}>0.05)$. Conclusions: The pedicled TRAM flap is an excellent alternative for reconstruction in breast cancer patients. It is not exempt from complications, with a tendency to get better cosmetic results in the non-smoker group.

Key words: Breast cancer, breast reconstruction, TRAM flap, cosmetic outcome, TRAM complications.

\section{Resumen}

El colgajo de recto abdominal con isla transversal de piel infraumbilical (TRAM) pediculado es ampliamente utilizado para la reconstrucción mamaria, sin embargo, no está exento de complicaciones. Entre los años 2004 y 2010 los autores realizaron reconstrucción mamaria post-mastectomía total en 52 pacientes con colgajos TRAM pediculados. Objetivo: Describir la demografía, resultados y complicaciones de las pacientes operadas por cáncer de mama y reconstruidas con colgajo TRAM. Evaluar la influencia del tabaquismo, la lateralidad y el momento de la reconstrucción en dichos resultados. Material y Método: Diseño retrospectivo, observacional y comparativo. Se revisaron las historias clínicas de las 52 pacientes operadas por los autores entre los años 2004-2010. Se excluyeron 4 casos por datos incompletos. Resultados: En las

*Recibido el 12 de enero de 2011 y aceptado para publicación el 11 de julio de 2011.

Correspondencia: Dr. Nicolás Pereira C.

Santos Dumont 999. Santiago, Chile.

nicolaspereirac@gmail.com 
48 pacientes estudiadas se realizaron 50 colgajos TRAM. La comorbilidad más frecuente fue el tabaquismo (27\%). La reconstrucción se realizó en un 74\% (37) de manera inmediata postmastectomía y en un 26\% (13) fue diferido. Se utilizó en un 78\% (39) TRAM ipsilateral, 18\% (9) contralateral y 4\% (2) bilateral. Sólo se observaron complicaciones menores; el resultado estético evaluado por la satisfacción del cirujano fue muy bueno o excelente en un $62 \%$ (31). El tabaquismo, momento de la reconstrucción y la lateralidad no influyeron en las complicaciones ni en el resultado estético $(p>0,05)$. Conclusiones: El colgajo TRAM pediculado es una excelente alternativa de reconstrucción en pacientes mastectomizadas. No está exento de complicaciones, con una tendencia a obtener mejores resultados estéticos en el grupo no fumador.

Palabras clave: Cáncer de mama, reconstrucción mamaria, colgajo TRAM, resultado estético, complicaciones TRAM.

\section{Introducción}

En cirugía oncológica de mama, se realiza mastectomía total cada vez que no se pueda practicar una cirugía conservadora. La mastectomía total es una cirugía que produce un impacto psicológico importante en las pacientes. La reconstrucción mamaria se ha instalado como una excelente alternativa para las pacientes que son sometidas a esta cirugía. Promueve un estado de bienestar y revierte muchas de las secuelas sicológicas y emocionales asociadas a la mastectomía ${ }^{1-3}$. Existen muchos procedimientos reconstructivos descritos, como los implantes de silicona, los implantes-expansores, el colgajo de músculo dorsal ancho más implante $\mathrm{y}$, desde hace casi tres décadas, la reconstrucción con colgajo miocutáneo de recto abdominal con isla transversal de piel infraumbilical (TRAM) ${ }^{4-7}$. Este último fue descrito en 1982 por Hartrampf et $\mathrm{al}^{8}$, desde entonces se ha convertido en el "caballito de batalla" en cuanto a la reconstrucción mamaria con tejido autólogo respecta. Durante los últimos años, se han utilizado técnicas microquirúrgicas utilizando éste y otros músculos, así como también colgajos perforantes como el de la arteria epigástrica inferior profunda.

El colgajo TRAM pediculado, ampliamente utilizado para la reconstrucción mamaria, no está exento de complicaciones 9 . Entre el 2004 y 2010 los autores G.I. y M.I. realizaron reconstrucciones mamarias post-mastectomía total en 52 pacientes con colgajos TRAM pediculados. El objetivo de nuestro trabajo es describir la demografía, resultados y complicaciones de las pacientes operadas por cáncer de mama y reconstruidas con colgajo TRAM en dos centros del área norte de la capital, y evaluar la influencia del tabaquismo, la lateralidad y el momento de la reconstrucción en los resultados.

\section{Material y Método}

\section{Grupo de estudio}

Se estudiaron todas las pacientes en los cuales se realizó reconstrucción mamaria post-mastectomía con colgajo TRAM pediculado por los autores M.I y G.I entre el período 2004-2010 en dos centros del área norte de Santiago; Complejo Hospitalario San José y Clínica Dávila. En ellos se revisaron los registros de la Unidad de Patología Mamaria y las historias clínicas de todas las pacientes para obtener información sobre datos demográficos, antecedentes del cáncer, detalles quirúrgicos, evolución postoperatoria y complicaciones detectadas durante la hospitalización y en los controles sucesivos. Así mismo, se revisaron archivos fotográficos de la evolución para complementar la información. Se incluyeron indistintamente pacientes en las cuales se practicó reconstrucción inmediata, diferida, uni o bilateral.

\section{Técnica quirúrgica}

En todas nuestras pacientes se utilizó el colgajo músculo-cutáneo de recto abdominal con isla cutánea transversa (TRAM). Están descritos distintos territorios cutáneos según su relación con las perforantes (Figura 1A); es así como el sector 1 es el territorio cutáneo situado sobre el músculo y su aponeurosis a utilizar. Esta zona es irrigada directamente por las perforantes y por lo tanto, tiene una muy buena vitalidad.

Puede utilizarse el recto ipsi o contralateral a la lesión. Levantamos el colgajo según la siguiente técnica (Figura 1):

- Diseño aproximado de los territorios cutáneos (Figura 1A).

- Incisión en la línea superior del colgajo a usar y disección cutánea hasta llegar al reborde costal. Superposición de este tejido que permite fijar el límite inferior del colgajo a usar, según la posibilidad de cierre.

- Levantamiento de las zonas 3 y 4 . La zona 4 la eliminamos generalmente por su irrigación deficiente.

- Disección de la zona 2 hasta 1 ó $2 \mathrm{~cm}$ por dentro del borde externo de la vaina del recto.

- Sección de la aponeurosis y levantamiento por disección roma digital del músculo y del pedículo vascular inferior previa sección. 


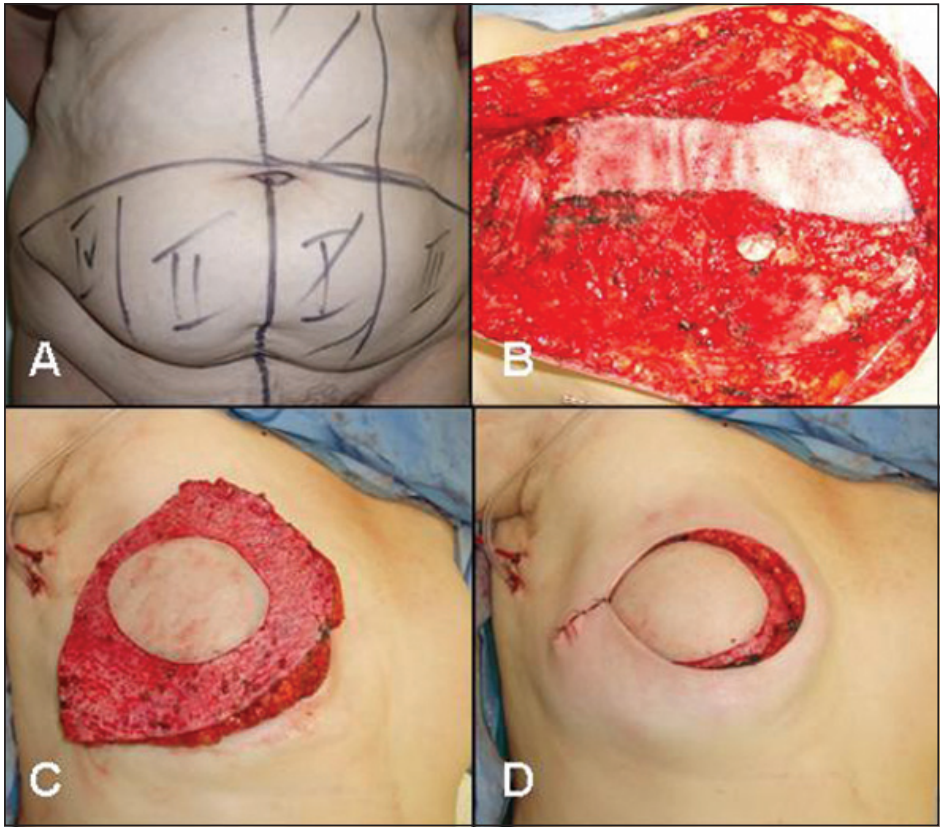

Figura 1. Técnica quirúrgica. A: Diseño de los territorios cutáneos. B: Reemplazo de defecto con malla de polipropileno. $\mathbf{C}$ y D: Se extirpa la piel sobrante y lleva el tejido celular subcutáneo a rellenar las zonas necesarias en el bolsillo de la neomama.
- Levantamiento del músculo llevando una franja central de aponeurosis de 1 ó $2 \mathrm{~cm}$. Reemplazo del defecto con malla de polipropileno (Figura 1B).

- Extirpación de la cicatriz torácica y formación de un bolsillo hacia cefálico y caudal que permita acomodar el colgajo (en reconstrucciones diferidas).

- Tunelización por sobre la vaina del recto para llevar el colgajo al sitio deseado.

- Acomodación del colgajo extirpando la piel sobrante (maniobra de Schwartzman) y llevando el tejido celular subcutáneo a rellenar las zonas necesarias (Figura 1C y D).

- Sutura de la pared abdominal por planos.

\section{Análisis de datos}

Se recopilaron datos de las historias clínicas de las pacientes en los dos centros mencionados. Se realizó análisis estadístico para evaluar la influencia de la lateralidad del colgajo (ipsi o contralateral), el momento de la reconstrucción (inmediata o diferida) y el tabaquismo, tanto sobre las complicaciones en el colgajo (sufrimiento, necrosis parcial, pérdida total, infección herida, seroma, necrosis grasa y estética deficiente) o en el sitio donante (sufrimiento, necrosis parcial, pérdida total, infección herida, seroma, hematoma, estética deficiente, defecto de cicatrización, hernia incisional), así como en el resultado estético, utilizando la prueba de $\chi^{2}$ en el programa computacional Stata 11.0. Se consideró estadísticamente significativo un valor $\mathrm{p}<0,05$. El resultado estético se evaluó de manera subjetiva por dos de los autores a través de controles presenciales y archivo fotográfico, clasificándose en cinco categorías (excelente, muy bueno, bueno, regular e inaceptable).

\section{Resultados}

\section{Características de los pacientes}

Se revisaron las historias clínicas de las 52 pacientes operadas por los autores entre el año 20042010. Se excluyeron 4 casos por datos incompletos. En las 48 pacientes estudiadas se realizaron 50 colgajos TRAM. El promedio de edad al diagnóstico de cáncer de mama fue 43,84 (rango 31-60) y al momento de la reconstrucción fue 44,7 años (rango 31-60). La comorbilidad más frecuente fue el tabaquismo $(27 \%)$ y el $68,7 \%$ tenía al menos un factor de riesgo para desarrollar cáncer de mama, siendo los antecedentes familiares el de mayor importancia $(29 \%)$.

Con respecto al estadio clínico, el $67 \%$ estaba entre IIA y IIB (Tabla 1). La histología más frecuente fue el tipo ductal infiltrante (68\%) (Tabla 2). En cuanto al momento de la reconstrucción, un $74 \%$ (37) se realizó de manera inmediata post-mastectomía y un $26 \%$ (13) fue diferido en un promedio de 42,7 meses. Se utilizó en un 78\% (39) TRAM ipsilateral, $18 \%$ (9) contralateral y $4 \%$ (2) bilateral.

Se reportaron 5 casos de recidiva post-mastectomía parcial primaria, que requirieron re-mastectomía 
y se reconstruyeron de manera inmediata luego de la segunda cirugía. En el período estudiado, hubo una mortalidad del 6,25\% (3 pacientes) por cáncer que correspondían a los casos con estadios más avanzados.

\section{Complicaciones y resultado estético}

Las complicaciones (Tabla 3) observadas se clasificaron dependiendo si eran originadas en el colgajo o si ocurrieron en el sitio donante. Con respecto a las complicaciones en el colgajo (Figura 2), se observó con mayor frecuencia la necrosis grasa (26\%), seguida del sufrimiento del colgajo (16\%). No se detectaron complicaciones mayores como la pérdida total del colgajo.

Tabla 1. Características de los pacientes

\begin{tabular}{|lcccc|}
\hline & \multicolumn{2}{c}{$\begin{array}{c}\text { Reconstrucción } \\
\text { inmediata } \\
(\mathbf{n = 3 7 )}\end{array}$} & $\begin{array}{c}\text { Reconstrucción } \\
\text { diferida } \\
(\mathbf{n = 1 3})\end{array}$ \\
& $\mathbf{n}$ & $\mathbf{\%}$ & $\mathbf{n}$ & $\mathbf{\%}$ \\
Edad promedio (años) & 44,7 & & 45,8 & \\
Tabaquismo & 9 & 24,3 & 4 & 30,7 \\
Estadio clínico & & & & \\
I & 1 & 2,7 & 1 & 7,6 \\
IIA & 12 & 32,4 & 5 & 38,4 \\
IIB & 11 & 29,7 & 6 & 46,1 \\
IIIA & 4 & 10,8 & 1 & 7,6 \\
IIIB & 8 & 21,6 & 0 & 0 \\
IV & 1 & 2,7 & 0 & 0 \\
\hline
\end{tabular}

Pacientes separadas según momento de la reconstrucción detallando características como edad, tabaquismo y estadio clínico en frecuencia absoluta y porcentajes.
En cuanto a las complicaciones en el sitio donante, la complicación que se observó con mayor frecuencia fue la necrosis parcial menor $(<2 \mathrm{~cm})$ del colgajo abdominal en un 14\%, involucrando en 4 casos al ombligo y en 3 casos la zona media de la herida abdominal. Tampoco se observaron complicaciones mayores en esta región.

Con respecto al resultado estético, éste fue evaluado de manera subjetiva por dos de los autores (G.I, M.I) a través de su grado de satisfacción, obteniéndose excelente en un 50\% (25) (Figura 3), muy bueno en $12 \%$ (6), bueno en $28 \%$ (14) y regular en $10 \%(5)$, sin resultados estéticos inaceptables.

Al analizar la influencia del momento de la reconstrucción (inmediato vs diferido) sobre las complicaciones y el resultado estético, éste no influyó en ninguna de las dos variables estudiadas no siendo estadísticamente significativas las diferencias $(\mathrm{p}=$ 0,856 y $\mathrm{p}=0,279$ respectivamente). Por otro lado, se evaluó la lateralidad del colgajo (ipsilateral vs contralateral) sobre las mismas variable, sin mostrar diferencias significativas ( $\mathrm{p}=0,56$ para las complicaciones y $\mathrm{p}=0,074$ para el resultado estético).

Por último se revisó la influencia del tabaquismo

Tabla 2. Tipo histológico

\begin{tabular}{|lcc|}
\hline Histología & n & \% \\
\hline Ductal in situ & 7 & 14 \\
Lobulillar in situ & 0 & 0 \\
Ductal infiltrante & 32 & 64 \\
Lobulillar infiltrante & 8 & 16 \\
\hline Ductolobulillar infiltrante & 3 & 6 \\
\hline
\end{tabular}

Frecuencias absolutas y porcentajes de los diferentes tipos histológicos encontrados en las pacientes mastectomizadas.

Tabla 3. Complicaciones del colgajo y sitio donante

\begin{tabular}{|lcclcc|}
\hline Abdomen & $\mathbf{n}$ & $\mathbf{\%}$ & Colgajo & n & \% \\
Sufrimiento & 3 & 6 & Sufrimiento & 8 & 16 \\
Necrosis parcial & 7 & 14 & Necrosis parcial & 4 & 8 \\
Pérdida total & 0 & 0 & Pérdida total & 0 & 0 \\
Infección herida & 1 & 2 & Infección herida & 2 & 4 \\
Seroma & 2 & 4 & Seroma & 13 & 26 \\
Hematoma & 1 & 2 & Necrosis grasa & 6 & 12 \\
Estética deficiente & 1 & 2 & Estética deficiente & & \\
Defecto de cicatrización & 2 & 4 & & & \\
\hline Hernia incisional & 3 & 6 & & & \\
\hline
\end{tabular}

Frecuencia y porcentaje de complicaciones tanto en el abdomen como en el colgajo TRAM. 
Tabla 4. Resultado estético según satisfacción del cirujano

\begin{tabular}{|lrc|}
\hline Resultado & n & \% \\
Excelente & 25 & 50 \\
Muy bueno & 6 & 12 \\
Bueno & 14 & 28 \\
Regular & 5 & 10 \\
Inaceptable & 0 & 0 \\
\hline
\end{tabular}

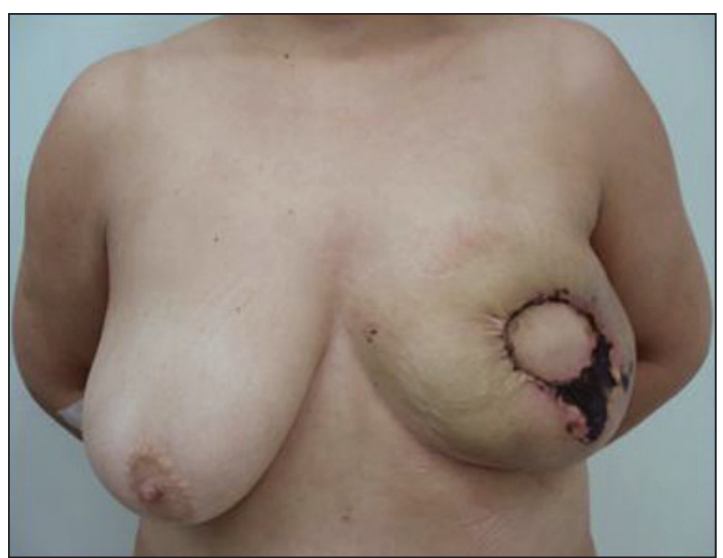

Figura 2. Necrosis parcial del colgajo receptor de la neomama. Se observa una necrosis parcial del colgajo que forma el bolsillo de la neomama. En esta paciente fue necesario realizar cirugía de reparación de esta complicación local. sobre las complicaciones y el resultado estético, no observándose diferencias de significación estadísticas $(p=0,313$ para las complicaciones y $p=0,057$ para el resultado estético).

\section{Discusión}

El método utilizado mayormente a nivel nacional e internacional para la reconstrucción mamaria es el colgajo TRAM ${ }^{10-12}$, técnica de elección en nuestro grupo para reconstruir a nuestras pacientes.

El componente familiar como factor de riesgo para desarrollar cáncer de mama se presenta de manera transversal en todas las pacientes, sin ser la excepción aquellas que se someten a reconstrucción mamaria. En nuestra muestra, al igual que en otros estudios $^{13}$, el carcinoma ductal es el que presenta una mayor incidencia, siendo el de tipo infiltrante el que fue intervenido en mayor número de casos. Al igual que en series internacionales, la mayor parte de nuestras pacientes operadas se encontraban en estadio clínico IIA y IIB de la American Joint Committee on Cancer (AJCC).

La reconstrucción mamaria se ha perfilado como una cirugía de rutina en las pacientes mastectomizadas, sin embargo, a pesar que el colgajo TRAM es el de mayor aplicación sigue siendo un procedimiento no exento de complicaciones aún en manos experimentadas. Se evidenció que hubo una considerable incidencia de necrosis grasa (26\%) en el colgajo, lo cual es comparable con estudios americanos que reportan $26,9 \%$ de necrosis grasa clínicamente detectable y $13,4 \%$ en mamografía ${ }^{14}$. Con respecto a otras complicaciones en el colgajo de mayor gravedad,

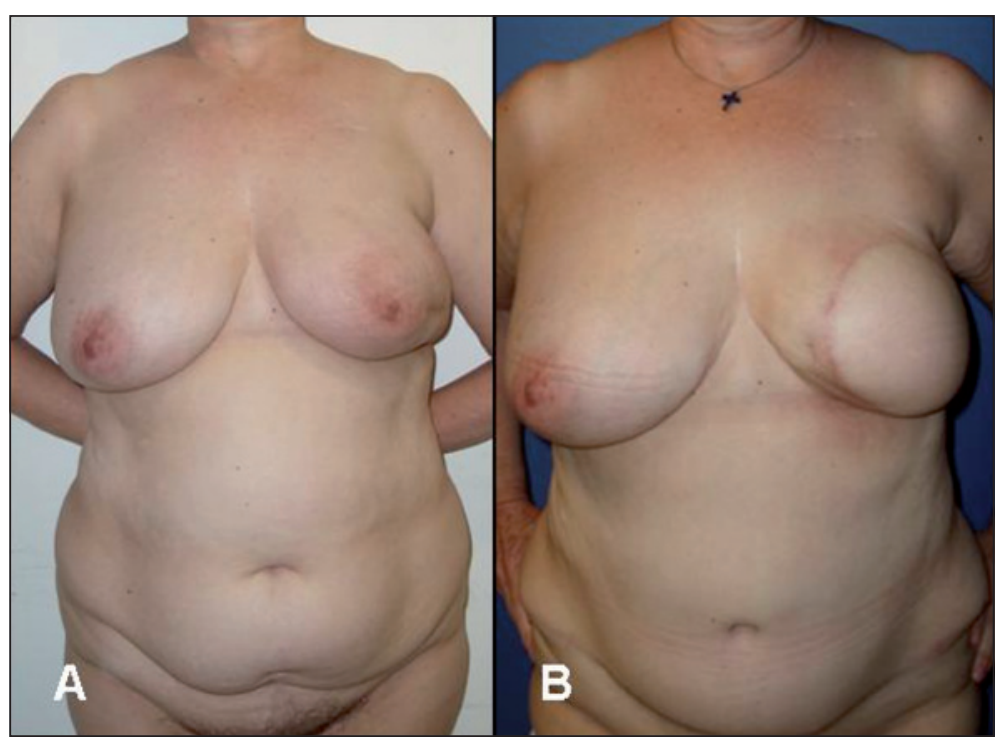

Figura 3. Imágenes pre y post operatorias de mastectomía total reconstruida. En estas imágenes se observa un "excelente" resultado estético, clasificado así por la evaluación subjetiva de los autores. 
como la pérdida total de éste, no se reportó en nuestra serie. Si bien, el sufrimiento y la necrosis parcial del colgajo ocurrieron en un pequeño grupo de pacientes, generalmente se manejaron con medidas conservadoras. En cuanto a las complicaciones del sitio donante, se observó una incidencia comparable con otros estudios ${ }^{15}$ y que la mayor parte de ellas se asociaba a sufrimiento y necrosis parcial del colgajo abdominal. Con respecto a esto, se debe hacer notar que esta complicación se presentó principalmente en relación al ombligo y a la zona media de la herida abdominal, lo cual nos sugiere que puede deberse a una mayor tensión en esta zona asociado a una irrigación deficiente en este territorio.

Lamentablemente, no existen disponibles instrumentos como BREAST-Q, validados en la población chilena para evaluar el resultado estético. Debido a lo anterior, el resultado estético fue evaluado de manera subjetiva a través de la satisfacción del equipo quirúrgico, lo cual puede constituir un sesgo. Probablemente una medición de manera estandarizada mediante instrumentos validados, podrían constituir una mejor aproximación. No obstante, los resultados obtenidos de esta manera nos reafirman al colgajo TRAM como una excelente alternativa de reconstrucción desde el punto de vista de resultados estéticos y se compara con otras casuísticas locales ${ }^{16}$.

No se observó relación estadísticamente significativa al comparar la influencia del momento de la reconstrucción (inmediata vs diferida), la lateralidad (ipsilateral vs contralateral) ni el hábito tabáquico, tanto en la incidencia de complicaciones como en los resultados estéticos. A pesar de lo anterior, la relación del hábito de fumar con el resultado estético nos sugiere que habría una influencia negativa por la tendencia que se observa en el análisis estadístico $(\mathrm{p}=0,057)$ que no alcanza a ser significativo; aún así, los datos son sugerentes de mejores resultados estéticos en el grupo no fumador. Una muestra mayor probablemente podría mostrar esa asociación. Sin embargo, el análisis estadístico tiene un rol exploratorio y no inferencial, ya que se trata de un estudio retrospectivo en el cual, por el diseño, no se realiza cálculo de tamaño muestral ni muestreo aleatorio. La conducta del equipo de autores es indicar la suspensión del hábito tabáquico al momento de su diagnóstico y advertir a las pacientes de los posibles eventos adversos relacionados con su mantención.

\section{Conclusiones}

El colgajo TRAM pediculado es una excelente alternativa de reconstrucción en pacientes sometidos a mastectomía total. Esta técnica no está exenta de complicaciones y el tabaquismo presentaría una influencia negativa sobre los resultados estéticos ya que se observó una tendencia a mejores resultados en las pacientes no fumadoras, sin influir en éstos el momento de la reconstrucción ni la lateralidad del colgajo.

\section{Referencias}

1. Asplund O, Korlof B. Late results following mastectomy for cancer and breast reconstruction. Scand J Plast Reconstr Surg. 1984;18:221-5.

2. Stevens LA, McGrath MH, Druss RG, Kister SJ, Gump FE, Forde KA. The psychological impact of immediate breast reconstruction for women with early breast cancer. Plast Reconstr Surg. 1984;73:619-28.

3. Kroll S. Immediate breast reconstruction: A review. Ann Chir Gynaecol. 1997;86:5-12.

4. Vasconez L, Grotting JC, Calderón W, Mathes SJ. The reconstruction of the breast. Where do we fall short? An evolution of ideas. Am J Surg. 1984;148:103-10.

5. Vasconez L, Lejours M, Gamboa M. Atlas of breast reconstruction. Philadelphia: JB Lippincott \& Co 1991.

6. Kroll SS. Why autologous tissue? Clin Plastic Surg. 1998;25:135-43.

7. Bostwick III J. Why I choose autogenous tissue in breast reconstruction. Clin Plastic Surg. 1994;21:165-75.

8. Hartrampf CR, Scheflan M, Black PW. Breast reconstruction with a transverse abdominal island flap. Plast Reconstr Surg. 1982;69:216-25.

9. Alderman AK, Wilkins EG, Myra Kim H, Lowery JC. Complications in postmastectomy breast reconstruction: Two-Year Results of the Michigan Breast Reconstruction Outcome Study. Plast Reconstr Surg. 2002; 109:2265-74.

10. Evans G, Kroll S. Choice of technique for reconstruction. Clin Plast Surg. 1998;25:311-6.

11. Villalón F. Reconstrucción mamaria con colgajo de recto abdominal. Rev Chil Cir. 1992;44:162-7.

12. Calderón O. Reconstrucción mamaria inmediata postmastectomía por cáncer. Rev Chil Cir. 1997;49:552-7.

13. Iglesias R, Cabello R, Fontbona M. Cirugía oncoplástica de la mama: Cómo mejorar la resecabilidad en cirugía conservadora asegurando la estética. Rev Chil Cir. 2009;61:433-7.

14. Kroll S, Gherardini G, Martin J. Fat Necrosis in Free and Pedicled TRAM Flaps. Plast Reconstr Surg. 1998;102:1502-7.

15. Petit JY, Rietjens M, Garusi C, Giraldo A, De Lorenzi F, Rey P, et al. Abdominal Complications and Sequelae after Breast Reconstruction with Pedicled TRAM Flap: Is There Still an Indication for Pedicled TRAM in the Year 2003? Plast Reconstr Surg 2003;112:1063-5.

16. Paredes H. Reconstrucción mamaria con colgajo miocutáneo de recto anterior. Rev Chil Cir. 1998;50:22-7. 